PROCEEDINGS OF THE

AMERICAN MATHEMATICAL SOCIETY

Volume 124, Number 12, December 1996, Pages 3767-3776

S 0002-9939(96)03526-5

\title{
SAMPLING IN A HILBERT SPACE
}

\author{
AHMED I. ZAYED
}

(Communicated by J. Marshall Ash)

\begin{abstract}
An analog of the Whittaker-Shannon-Kotel'nikov sampling theorem is derived for functions with values in a separable Hilbert space. The proof uses the concept of frames and frame operators in a Hilbert space. One of the consequences of this theorem is that it allows us to derive sampling theorems associated with boundary-value problems and some homogeneous integral equations, which in turn gives us a generalization of another sampling theorem by Kramer.
\end{abstract}

\section{INTRODUCTION}

Since its introduction in communication engineering by Shannon in 1948 [12], the Whittaker-Shannon-Kotel'nikov sampling theorem has played an important role in both mathematics and electrical engineering. Briefly, the theorem says that if a signal (function) of time, $t$, is limited in its bandwidth to $W$ cycles per second, it is completely determined by its values at a series of discrete points spaced $1 / 2 \mathrm{~W}$ seconds apart and can be recovered at any time $t$ by using its values at that discrete set of points. Mathematically, this can be rephrased as follows: If $f(t)$ is a function band-limited to $[-2 \pi W, 2 \pi W]$, i.e.,

$$
f(t)=\frac{1}{\sqrt{2 \pi}} \int_{-\sigma}^{\sigma} F(\omega) e^{i t \omega} d \omega,
$$

for some $F \in L^{2}[-\sigma, \sigma]$, where $\sigma=2 \pi w$, then it can be reconstructed from its samples at the points $t_{k}=k \pi / \sigma, k=0, \pm 1, \pm 2, \ldots$, via the formula

$$
f(t)=\sum_{k=-\infty}^{\infty} f\left(t_{k}\right) \frac{\sin \sigma\left(t-t_{k}\right)}{\sigma\left(t-t_{k}\right)}, \quad t \in \mathfrak{R}
$$

where the series converges absolutely and uniformly on compact sets of the real line $\Re$.

This theorem has been generalized in many different ways. In one direction, the equally spaced points $\left\{t_{k}\right\}_{k \in \mathcal{Z}}(\mathcal{Z}$ is the set of integers) are replaced by nonuniformly spaced points leading to the following generalization by Paley and Wiener $[11$, p. 115]:

Received by the editors May 30, 1994 and, in revised form, June 5, 1995.

1991 Mathematics Subject Classification. Primary 41A05, 41A35; Secondary 47A58.

Key words and phrases. Shannon's sampling theorem, interpolation and approximation in a Hilbert space, frames and frame operators.

(c)1996 American Mathematical Society 
Let $\left\{t_{k}\right\}_{k \in \mathcal{Z}}$ be a sequence of real numbers such that

$$
\sup _{k \in \mathcal{Z}}\left|t_{k}-\frac{k \pi}{\sigma}\right|<\frac{\pi}{4 \sigma},
$$

and let $P(t)$ be the entire function defined by

$$
P(t)=\left(t-t_{0}\right) \prod_{k=1}^{\infty}\left(1-\frac{t}{t_{k}}\right)\left(1-\frac{t}{t_{-k}}\right) .
$$

Then for any $f$ in the form (1.1), we have

$$
f(t)=\sum_{k=-\infty}^{\infty} f\left(t_{k}\right) \frac{P(t)}{\left(t-t_{k}\right) P^{\prime}\left(t_{k}\right)}
$$

with the series being absolutely and uniformly convergent on compact sets. If $t_{k}=k \pi / \sigma$, then $P(t)$ reduces to $\sin (\sigma t) / \sigma$ and (1.4) reduces to (1.2).

In another direction, the kernel function, $e^{i t \omega}$, in (1.1) is replaced by a more general kernel $K(\omega, t)$ leading to the following generalization by Kramer [8]: Let $K(x, t)$ be a continuous function in $t$ such that as a function of $x, K(x, t) \in L^{2}(I)$ for every real number $t$, where $I=[a, b],-\infty<a<b<\infty$. Assume that there exists a sequence of real numbers $\left\{t_{k}\right\}_{k \in \mathcal{Z}}$ such that $\left\{K\left(x, t_{k}\right)\right\}_{k \in \mathcal{Z}}$ is a complete orthogonal family of functions in $L^{2}(I)$. Then for any $f$ of the form

$$
f(t)=\int_{a}^{b} K(x, t) F(x) d x,
$$

where $F \in L^{2}(I)$, we have

$$
f(t)=\sum_{k=-\infty}^{\infty} f\left(t_{k}\right) S_{k}^{*}(t)
$$

with

$$
S_{k}^{*}(t)=\frac{\int_{a}^{b} K(x, t) \overline{K\left(x, t_{k}\right)} d x}{\int_{a}^{b}\left|K\left(x, t_{k}\right)\right|^{2} d x} .
$$

If $I=[-\sigma, \sigma], t_{k}=k \pi / \sigma$, and $K(x, t)=e^{i x t}$, it is easy to see that

$$
S_{k}^{*}(t)=\frac{\sin \sigma\left(t-t_{k}\right)}{\sigma\left(t-t_{k}\right)},
$$

and hence (1.6) reduces to (1.2).

One way to generate the kernel $K(x, t)$ and the sampling points $\left\{t_{k}\right\}_{k \in \mathcal{Z}}$ is to consider the regular Sturm-Liouville boundary-value problem:

$$
\begin{gathered}
-y^{\prime \prime}+q(x) y=t y, \quad x \in I=[a, b], \\
y(a) \cos \alpha+y^{\prime}(a) \sin \alpha=0, \\
y(b) \cos \beta+y^{\prime}(b) \sin \beta=0,
\end{gathered}
$$

where $q$ is continuous on $I$. Then take $K(x, t)$ to be the solution of the differential equation (1.7) and the initial condition (1.8) (or the solution of (1.7) and (1.9)), and take the sampling points $\left\{t_{k}\right\}_{k \in \mathcal{Z}}$ to be the eigenvalues of the problem since the eigenfunctions $\left\{K\left(x, t_{k}\right)\right\}_{t \in \mathcal{Z}}$ form a complete orthogonal family in $L^{2}(I)$. 
Although it is theoretically feasible to extend this procedure to more general self-adjoint boundary-value problems associated with $n$th order differential operators, in practice this does not always work since the existence of one single function $K(x, t)$, which generates all the eigenfunctions of the problem when the parameter $t$ is replaced by the eigenvalues, is not always guaranteed. For example, the eigenfunctions, $\cos 2 n x$ and $\sin 2 n x$, of the boundary-value problem

$$
\begin{gathered}
-y^{\prime \prime}=t y, \quad x \in[0, \pi], \\
y(0)=y(\pi) \quad \text { and } \quad y^{\prime}(0)=y^{\prime}(\pi),
\end{gathered}
$$

are not generated by one single real-valued function.

One possibility to circumvent this problem is to use the Green's function method described in [15]. For many self-adjoint boundary-value problems, the Green's functions can be written in the form

$$
G(x, y, \lambda)=\sum_{n=1}^{\infty} \frac{\phi_{n}(x) \phi_{n}(y)}{\lambda-\lambda_{n}}
$$

where $\left\{\lambda_{n}\right\}_{n=1}^{\infty}$ are the eigenvalues and $\left\{\phi_{n}\right\}_{n=1}^{\infty}$ are the corresponding eigenfunctions. The Green's function method can also be used to derive sampling theorems associated with homogeneous Fredholm integral equations of the second kind. For more on Shannon's sampling theorem, its generalizations, and connection with boundary-value problems, see [14].

The aim of this paper is to generalize some of the above results to derive a sampling theorem for vector-valued functions. These functions take values in a separable Hilbert space $\mathcal{H}$. One of the interesting ramifications of this generalization is that it allows us to obtain sampling theorems associated with boundary-value problems and integral equations without restricting the sampling points to be the eigenvalues of the corresponding problem. In fact, the sampling points will be arbitrary, except for a restriction on their growth rate.

The idea of using Hilbert space concepts in sampling theory goes back to F. Beutler [4], [5] and K. Yao [13]. More recently, J. Benedetto [1], [2] and J. Benedetto and $\mathrm{W}$. Heller [3] used the concept of frames in a Hilbert space to derive sampling theorems for band-limited functions. In this paper we also derive our sampling theorem by using the concept of frames though in a different context than that in [1]-[3].

\section{Preliminaries}

Throughout the rest of this article the sets of complex and real numbers will be denoted by $\mathbb{C}$ and $\mathfrak{R}$ respectively and $\mathcal{H}$ will denote a separable Hilbert space with inner product $\langle$,$\rangle and norm \|x\|=\sqrt{\langle x, x\rangle}$ for all $x \in \mathcal{H}$. The Fourier transform of a function $f(t)$ is defined as

$$
\hat{f}(\omega)=\frac{1}{\sqrt{2 \pi}} \int_{-\infty}^{\infty} f(t) e^{i t \omega} d t
$$

so that the inverse transform is given by

$$
f(t)=\frac{1}{\sqrt{2 \pi}} \int_{-\infty}^{\infty} \hat{f}(\omega) e^{-i t \omega} d \omega
$$

provided that the integrals exist. Let $B_{\sigma}^{2}$ denote the class of all entire functions $f$ of exponential type at most $\sigma$ belonging to $L^{2}(\mathfrak{R})$ when restricted to the real axis; 
that is, $f \in B_{\sigma}^{2}$ if and only if

$$
|f(z)| \leq \sup _{x \in \Re}|f(x)| \exp (\sigma|y|),
$$

where $z=x+i y$, and

$$
\int_{-\infty}^{\infty}|f(x)|^{2} d x<\infty
$$

The well-known Paley-Wiener Theorem [11, p. 13] asserts that

$$
f \in B_{\sigma}^{2}(\sigma>0) \text { if and only if } f(t)=\frac{1}{\sqrt{2 \pi}} \int_{-\sigma}^{\sigma} F(\omega) e^{i t \omega} d \omega,
$$

for some $F \in L^{2}[-\sigma, \sigma]$. The class $B_{\sigma}^{2}$ is usually called the Paley-Wiener class of entire functions.

Let $\mathcal{G}=\left\{g_{n}\right\}$ be a sequence in $\mathcal{H}$. We say that $\mathcal{G}$ is a frame if there exist two numbers $A, B>0$ such that for every $f \in \mathcal{H}$,

$$
A\|f\|^{2} \leq \sum_{n}\left|\left\langle f, g_{n}\right\rangle\right|^{2} \leq B\|f\|^{2} .
$$

The two numbers $A$ and $B$ are called the frame bounds. The frame is said to be tight if $A=B$ and is exact if it ceases to be a frame whenever any single element is deleted from the frame. Frames are complete since if $\left\langle f, g_{n}\right\rangle=0$ for all $n$, then $\|f\|=0$ and consequently $f=0 . \mathcal{G}$ is said to be bounded if there exist two nonnegative numbers $C$ and $D$ such that $C \leq\left\|g_{n}\right\| \leq D$ for all $n$. It is known [7] that a frame is exact if and only if it is a bounded and unconditional basis. A basis $\mathcal{G}$ is said to be unconditional if

$$
\sum_{n} c_{n} g_{n} \in \mathcal{H} \quad \text { implies that } \sum\left|c_{n}\right| g_{n} \in \mathcal{H}
$$

With every frame $\mathcal{G}$, we associate a frame operator $S$ defined by

$$
S f=\sum_{n}\left\langle f, g_{n}\right\rangle g_{n}
$$

It can be shown [7] that $S$ is a bounded linear operator on $\mathcal{H}$ with $A I \leq S \leq B I$, and that $S$ is invertible, where $A I \leq S \leq B I$ means $A\langle x, x\rangle \leq\langle S x, x\rangle \leq B\langle x, x\rangle$ for all $x \in \mathcal{H}$. The inverse frame operator $S^{-1}$ has the following properties:

(1) $B^{-1} I \leq S^{-1} \leq A^{-1} I$,

(2) $\left\{S^{-1} g_{n}\right\}$ is also a frame with frame bounds $B^{-1}$ and $A^{-1}$.

\section{THE MAIN RESUlT}

Let $\left\{\lambda_{n}\right\}_{n=1}^{\infty}$ be a sequence of complex numbers, none of which is zero, with the point at infinity as its only limit point. The convergence exponent $\tau$ of the sequence $\left\{\lambda_{n}\right\}_{n=1}^{\infty}$ is defined as

$$
\tau=\inf \left\{\alpha \in \mathfrak{R}: \sum_{n=1}^{\infty} \frac{1}{\left|\lambda_{n}\right|^{\alpha}}<\infty\right\} .
$$

Let us further assume that $\left\{\lambda_{n}\right\}_{n=1}^{\infty}$ has finite convergence exponent, i.e., $0 \leq \tau<$ $\infty$. Let $p$ denote the smallest positive integer such that $\sum_{n=1}^{\infty} 1 /\left|\lambda_{n}\right|^{p+1}$ converges. 
Let

$P(\lambda)=\left\{\begin{array}{l}\prod_{n=1}^{\infty}\left(1-\frac{\lambda}{\lambda_{n}}\right) \exp \left[\left(\frac{\lambda}{\lambda_{n}}\right)+\frac{1}{2}\left(\frac{\lambda}{\lambda_{n}}\right)^{2}+\cdots+\frac{1}{p}\left(\frac{\lambda}{\lambda_{n}}\right)^{p}\right] \quad \text { if } p=1,2, \ldots, \\ \prod_{n=1}^{\infty}\left(1-\frac{\lambda}{\lambda_{n}}\right) \quad \text { if } p=0 .\end{array}\right.$

We may include zero as one of the terms of the sequence $\left\{\lambda_{n}\right\}$ and in this case we will denote it by $\lambda_{0}$ and redefine $P(\lambda)$ as

$P(\lambda)=\left\{\begin{array}{l}\lambda \prod_{n=1}^{\infty}\left(1-\frac{\lambda}{\lambda_{n}}\right) \exp \left[\left(\frac{\lambda}{\lambda_{n}}\right)+\frac{1}{2}\left(\frac{\lambda}{\lambda_{n}}\right)^{2}+\cdots+\frac{1}{p}\left(\frac{\lambda}{\lambda_{n}}\right)^{p}\right] \quad \text { if } p=1,2, \ldots, \\ \lambda \prod_{n=1}^{\infty}\left(1-\frac{\lambda}{\lambda_{n}}\right) \quad \text { if } p=0 .\end{array}\right.$

It can be shown that $P(\lambda)$ is an entire function in $\lambda$ of order equal to $\tau$ [6].

Let $\left\{g_{n}\right\}_{n=1}^{\infty}$ be a frame in a separable Hilbert space $\mathcal{H}$ and let $S$ be its frame operator. We shall denote the dual frame $\left\{S^{-1} g_{n}\right\}_{n=1}^{\infty}$ by $\left\{g^{*}\right\}_{n=1}^{\infty}$. If $\left\{g_{n}\right\}_{n=1}^{\infty}$ is exact, $\left\{g_{n}\right\}_{n=1}^{\infty}$ and $\left\{g_{n}^{*}\right\}_{n=1}^{\infty}$ are biorthonormal; that is, [7]

$$
\left\langle g_{m}, g_{n}^{*}\right\rangle=\delta_{m, n}= \begin{cases}0 & \text { if } m \neq n \\ 1 & \text { if } m=n\end{cases}
$$

For each fixed $\lambda \neq \lambda_{1}, \lambda_{2}, \ldots$, define the operator

$$
L_{\lambda}=P(\lambda) \sum_{n=1}^{\infty} \frac{\left\langle\cdot, g_{n}\right\rangle}{\left(\lambda-\lambda_{n}\right)} g_{n}^{*}
$$

on $\mathcal{H}$ in the usual way, i.e.,

$$
L_{\lambda} f=P(\lambda) \sum_{n=1}^{\infty} \frac{\left\langle f, g_{n}\right\rangle}{\left(\lambda-\lambda_{n}\right)} g_{n}^{*}, \quad f \in \mathcal{H},
$$

and for $\lambda=\lambda_{k}, k=1,2, \ldots$, define

$$
L_{\lambda_{k}}=P^{\prime}\left(\lambda_{k}\right)\left\langle\cdot, g_{k}\right\rangle g_{k}^{*} .
$$

For fixed $f \in \mathcal{H}$, define $F: \mathbb{C} \rightarrow \mathcal{H}$ by

$$
F(\lambda)=L_{\lambda} f
$$

Now we state and prove our sampling theorem.

Theorem 1. (1) For each fixed $\lambda \in \mathbb{C}, L_{\lambda}$ is a bounded linear operator on $\mathcal{H}$.

(2) If $K$ is a compact subset of the complex $\lambda$-plane, then the set of operators $\left\{L_{\lambda}\right\}_{\lambda \in K}$ is uniformly bounded.

(3) $F(\lambda)$ is a continuous vector-valued function that is completely determined by its values $\left\{F\left(\lambda_{n}\right)\right\}_{n=1}^{\infty}$ and can be reconstructed from these values according to the formula

$$
F(\lambda)=\sum_{n=1}^{\infty} \frac{P(\lambda)}{\left(\lambda-\lambda_{n}\right) P^{\prime}\left(\lambda_{n}\right)} F\left(\lambda_{n}\right) .
$$

Proof. The linearity of $L_{\lambda}$ is trivial once we show that $L_{\lambda}$ is well defined. We prove (1) and (2) together, but first let us recall that for any $\eta \in \mathcal{H},\|\eta\|$ can be given by $\|\eta\|=\sup _{\|h\|=1}|\langle\eta, h\rangle|$. Let

$$
S_{m, \lambda} f=P(\lambda) \sum_{k=1}^{m} \frac{\left\langle f, g_{k}\right\rangle}{\left(\lambda-\lambda_{k}\right)} g_{k}^{*}
$$


Then for $1 \leq m \leq n$ and by the Cauchy-Schwarz inequality, we have

$$
\begin{aligned}
\left\|S_{m, \lambda} f-S_{n, \lambda} f\right\|^{2} & =\sup _{\|h\|=1}\left|\left\langle S_{m, \lambda} f-S_{n, \lambda} f, h\right\rangle\right|^{2} \\
& =\sup _{\|h\|=1}\left|P(\lambda) \sum_{k=m+1}^{n} \frac{\left\langle f, g_{k}\right\rangle}{\left(\lambda-\lambda_{k}\right)}\left\langle g_{k}^{*}, h\right\rangle\right|^{2} \\
& \leq|P(\lambda)|^{2} \sup _{\|h\|=1}\left(\sum_{k=m+1}^{n} \frac{\left|\left\langle f, g_{k}\right\rangle\right|^{2}}{\left|\lambda-\lambda_{k}\right|^{2}}\right)\left(\sum_{k=m+1}^{n}\left|\left\langle g_{k}^{*}, h\right\rangle\right|^{2}\right) .
\end{aligned}
$$

Since $\left\{g_{k}^{*}\right\}_{k=1}^{\infty}$ is a frame with frame bounds $B^{-1}$ and $A^{-1}$, it follows that

$$
\left\|S_{m, \lambda} f-S_{n, \lambda} f\right\|^{2} \leq|P(\lambda)|^{2}\left(\sum_{k=m+1}^{n} \frac{\left|\left\langle f, g_{k}\right\rangle\right|^{2}}{\left|\lambda-\lambda_{k}\right|^{2}}\right) A^{-1} .
$$

Let $K$ be a compact subset of the complex $\lambda$-plane and $\widetilde{\Lambda}=\left\{\lambda_{i_{1}}, \ldots, \lambda_{i_{q}}\right\}$ be the set of $\lambda_{i}$ 's that lie inside $K$. Let us also denote the sequence $\left\{\lambda_{n}\right\}_{n=1}^{\infty}$ by $\Lambda$ and the distance between $K$ and $\Lambda-\tilde{\Lambda}$ by $\delta$. Then for any $\lambda \in K$ and $\lambda_{k} \in \Lambda-\tilde{\Lambda}$ we have

$$
\sup _{\lambda \in K}\left|\frac{P(\lambda)}{\lambda-\lambda_{k}}\right| \leq \frac{1}{\delta} \sup _{\lambda \in K}|P(\lambda)|=\frac{1}{\delta}\|P\|_{K},
$$

where $\|P\|_{K}=\sup _{\lambda \in K}|P(\lambda)|$. Set

$$
h_{i}(\lambda)=\frac{P(\lambda)}{\lambda-\lambda_{i}}, \quad i=i_{1}, i_{2}, \ldots, i_{q} .
$$

Clearly, $h_{i}$ is an analytic function except possibly at $\lambda=\lambda_{i}$, but since $P$ has a zero at $\lambda=\lambda_{i}, h_{i}$ is actually an entire function; therefore, $\max _{\lambda \in K}\left|h_{i}(\lambda)\right|=\left\|h_{i}\right\|_{K}$ is finite. Let $C=\max \left\{\left\|h_{i_{1}}\right\|_{K}, \ldots,\left\|h_{i_{q}}\right\|_{K}\right\}$, and $C(K)=\max \left\{C,\|P\|_{K} / \delta\right\}$. By combining this and (3.3), we obtain

$$
\left\|S_{m, \lambda} f-S_{n, \lambda} f\right\|^{2}<C^{2}(K)\left(\sum_{k=m+1}^{n}\left|\left\langle f, g_{k}\right\rangle\right|^{2}\right) A^{-1} \rightarrow 0
$$

as $m, n \rightarrow \infty$. Thus, $\left\{S_{m, \lambda} f\right\}_{m=1}^{\infty}$ is a Cauchy sequence and hence $\lim _{m \rightarrow \infty} S_{m, \lambda} f$ $=L_{\lambda} f$. By letting $n \rightarrow \infty$ in (3.4), it follows that $S_{m, \lambda}$ converges to $L_{\lambda} f$ uniformly on compact subsets of the complex $\lambda$-plane.

Repeating the above argument once more leads to

$$
\begin{aligned}
\|F(\lambda)\|^{2} & =\left\|L_{\lambda} f\right\|^{2}=\sup _{\|h\|=1}\left|\left\langle L_{\lambda} f, h\right\rangle\right|^{2} \\
& =|P(\lambda)|^{2} \sup _{\|h\|=1}\left(\sum_{k=1}^{\infty} \frac{\left|\left\langle f, g_{k}\right\rangle\right|^{2}}{\left|\lambda-\lambda_{k}\right|^{2}}\right)\left(\sum_{k=1}^{\infty}\left|\left\langle g_{k}^{*}, h\right\rangle\right|^{2}\right) \\
& \leq A^{-1} C^{2}(K)\left(\sum_{k=1}^{\infty}\left|\left\langle f, g_{k}\right\rangle\right|^{2}\right) \leq A^{-1} B C^{2}(K)\|f\|^{2},
\end{aligned}
$$

which shows that for fixed $\lambda, L_{\lambda}$ is a continuous linear operator on $\mathcal{H}$. In fact, Eq. (3.5) also shows that the family of operators $\left\{L_{\lambda}\right\}_{\lambda \in K}$ is uniformly bounded.

Now we prove part (3). From (1), it follows that $F(\lambda)$ is well defined. Now we show that it is continuous. Since $P(\lambda)$ is an entire function, it suffices to show that $G(\lambda)=(1 / P(\lambda)) F(\lambda)$ is continuous. Let $\lambda^{*} \in \mathbb{C}-\Lambda$ and denote the distance 
between $\lambda^{*}$ and $\Lambda$ by $2 \delta$. Let $D_{\delta}\left(\lambda^{*}\right)=\left\{\lambda:\left|\lambda-\lambda^{*}\right| \leq \delta\right\}$ be the closed disc with center $\lambda^{*}$ and radius $\delta$. For any $\lambda \in D_{\delta}\left(\lambda^{*}\right)$, we have

$$
\begin{aligned}
\left\|G(\lambda)-G\left(\lambda^{*}\right)\right\|^{2} & =\sup _{\|h\|=1}\left|\left\langle G(\lambda)-G\left(\lambda^{*}\right), h\right\rangle\right|^{2} \\
& \leq \sup _{\|h\|=1}\left(\sum_{k=1}^{\infty}\left(\frac{\left|\lambda^{*}-\lambda\right|}{\left|\lambda-\lambda_{k}\right|\left|\lambda^{*}-\lambda_{k}\right|}\right)^{2}\left|\left\langle f, g_{k}\right\rangle\right|^{2}\right)\left(\sum_{k=1}^{\infty}\left|\left\langle g_{k}^{*}, h\right\rangle\right|^{2}\right) \\
& \leq A^{-1}\left|\lambda^{*}-\lambda\right|^{2} \sum_{k=1}^{\infty}\left(\frac{1}{\left|\lambda-\lambda_{k}\right|\left|\lambda^{*}-\lambda_{k}\right|}\right)^{2}\left|\left\langle f, g_{k}\right\rangle\right|^{2} \\
& \leq A^{-1} \delta^{-4} B\|f\|^{2}\left|\lambda^{*}-\lambda\right|^{2},
\end{aligned}
$$

or

$$
\left\|G(\lambda)-G\left(\lambda^{*}\right)\right\| \leq(B / A)^{1 / 2} \frac{\|f\|}{\delta^{2}}\left|\lambda^{*}-\lambda\right| \rightarrow 0
$$

as $\lambda \rightarrow \lambda^{*}$.

Since $F(\lambda)$ is continuous for each $\lambda \in \mathbb{C}-\Lambda$ and

$$
\lim _{\lambda \rightarrow \lambda_{n}} F(\lambda)=F\left(\lambda_{n}\right)=P^{\prime}\left(\lambda_{n}\right)\left\langle f, g_{n}\right\rangle g_{n}^{*},
$$

$F$ is continuous everywhere. Finally, Eq. (3.2) follows from (3.1) and (3.6).

Corollary 2. Let $L$ be a one-one, self-adjoint compact operator on $\mathcal{H}$ and $\left\{g_{n}\right\}_{n=1}^{\infty}$ be its normalized eigenvectors. For any sequence $\left\{\lambda_{n}\right\}_{n=1}^{\infty}$ satisfying the assumption of Theorem 1, define $L_{\lambda}$ as before. Then for any $f \in \mathcal{H}$,

$$
F(\lambda)=L_{\lambda} f=\sum_{k=1}^{\infty} \frac{P(\lambda)}{\left(\lambda-\lambda_{k}\right) P^{\prime}\left(\lambda_{k}\right)} F\left(\lambda_{k}\right) .
$$

Proof. The eigenvectors of $L$ form an orthonormal basis, hence they form an exact, tight frame with frame bounds $=1$ and $g_{n}^{*}=g_{n}$.

As a special case, let $\mathcal{H}=L^{2}(I)$, where $I=[a, b],-\infty<a<b<\infty$, and

$$
(L f)(x)=\int_{a}^{b} K(x, \zeta) f(\zeta) d \zeta, \quad f \in L^{2}(I) .
$$

If $K$ is real, symmetric and in $L^{2}(Q)$, where $Q=I \times I$, then $L$ is a self-adjoint compact operator on $L^{2}(I)$. In addition, if the equation

$$
\int_{a}^{b} K(x, \zeta) f(\zeta) d \zeta=0
$$

has only the trivial solution $f=0$, then the eigenfunctions $\left\{g_{n}\right\}_{n=1}^{\infty}$ of $L$ form an orthonormal basis for $L^{2}(I)$, and we have the following sampling theorem: For any sequence of numbers $\left\{\lambda_{n}\right\}_{n=1}^{\infty}$ satisfying the assumption of Theorem 1 if

$$
F(\lambda)=\int_{a}^{b} f(x) R(x, \zeta, \lambda) d x,
$$

where

$$
R(x, \zeta, \lambda)=P(\lambda) \sum_{n=1}^{\infty} \frac{g_{n}(x) g_{n}(\zeta)}{\left(\lambda-\lambda_{n}\right)}
$$


$\zeta$ is a fixed point in $I$, then

$$
F(\lambda)=\sum_{n=1}^{\infty} F\left(\lambda_{n}\right) \frac{P(\lambda)}{\left(\lambda-\lambda_{n}\right) P^{\prime}\left(\lambda_{n}\right)} .
$$

When the $\lambda_{n}$ 's are taken as the eigenvalues of $L, R / P$ becomes the resolvent associated with the integral equation and in the case of self-adjoint boundary-value problems it becomes the Green's function of the problem; see (1.10).

The assumption that $L$ is self-adjoint can be replaced by the assumption that the eigenvectors of $L$ form an exact frame. This is the case for example when $L$ is associated with certain non-self-adjoint boundary-value problems; see [15].

\section{INVERSION FORMULA}

In this section we derive an inversion formula for the vector-valued function $F(\lambda)$ introduced in Theorem 1 by using a Bochner integral of $F$. To this end, we need to restrict the growth rate of the sequence $\left\{\lambda_{n}\right\}_{n=1}^{\infty}$ and require it to have $\pm \infty$ as its only limit points. Therefore, without loss of generality, it is more appropriate to use the integers as the index set for the sequence instead of the natural numbers.

Theorem 3. Let $\left\{\lambda_{n}\right\}_{n \in \mathcal{Z}}$ be a sequence of real numbers having no finite limit point such that

$$
\sup _{n \in \mathcal{Z}}\left|\lambda_{n}-(n \pi / \sigma)\right|<\frac{\pi}{4 \sigma}, \quad \sigma>0,
$$

and let

$$
P(\lambda)=\left(\lambda-\lambda_{0}\right) \prod_{n=1}^{\infty}\left(1-\frac{\lambda}{\lambda_{n}}\right)\left(1-\frac{\lambda}{\lambda_{-n}}\right),
$$

with

$$
\int_{-\infty}^{\infty}\left|\frac{P(\lambda)}{\lambda-\lambda_{n}}\right|^{2} d \lambda \leq D<\infty \quad \text { for all } n
$$

Then if

$$
F(\lambda)=P(\lambda) \sum_{n=-\infty}^{\infty} \frac{\left\langle f, g_{n}\right\rangle}{\left(\lambda-\lambda_{n}\right)} g_{n}^{*}, \quad f \in \mathcal{H},
$$

where $\left\{g_{n}\right\}_{n \in \mathcal{Z}}$ and $\left\{g_{n}^{*}\right\}_{n \in \mathcal{Z}}$ have the same meaning as before, then

$$
f=\lim _{N \rightarrow \infty} \int_{-\infty}^{\infty} F(\lambda) K_{N}(\lambda) d \lambda,
$$

where

$$
K_{N}(\lambda)=\frac{1}{2 \pi} \sum_{k=-N}^{N} \frac{B_{k}(\lambda)}{P^{\prime}\left(\lambda_{k}\right)}
$$

and

$$
B_{k}(\lambda)=\int_{-\sigma}^{\sigma} e^{i \lambda_{k} x} e^{i \lambda x} d x
$$


Proof. We infer from Theorem 18, p. 48 and Lemma 16.2, p. 57 in [9] that there exists a sequence of functions $\left\{h_{n}(x)\right\}_{n \in \mathcal{Z}}$ such that

$$
\frac{P(\lambda)}{\left(\lambda-\lambda_{n}\right) P^{\prime}\left(\lambda_{n}\right)}=\int_{-\sigma}^{\sigma} h_{n}(x) e^{i \lambda x} d x
$$

and

$$
\int_{-\sigma}^{\sigma} h_{n}(x) e^{i \lambda_{m} x} d x= \begin{cases}0 & \text { if } n \neq m \\ 1 & \text { if } n=m .\end{cases}
$$

Set

$$
B_{n}(\lambda)=\int_{-\sigma}^{\sigma} e^{i \lambda_{n} x} e^{-i \lambda x} d x
$$

hence

$$
e^{i \lambda_{n} x} \chi_{[-\sigma, \sigma]}(x)=\frac{1}{2 \pi} \int_{-\infty}^{\infty} B_{n}(\lambda) e^{i \lambda x} d \lambda,
$$

where $\chi_{[-\sigma, \sigma]}$ is the characteristic function of $[-\sigma, \sigma]$ and the integral converges in the sense of $L^{2}$. Let

$$
K_{N}(\lambda)=\frac{1}{2 \pi} \sum_{k=-N}^{N} \frac{B_{k}(\lambda)}{P^{\prime}\left(\lambda_{k}\right)}
$$

From (4.2)-(4.4) and Parseval's equality, we have

$$
\frac{1}{2 \pi} \int_{-\infty}^{\infty} \frac{P(\lambda) B_{k}(\lambda)}{\left(\lambda-\lambda_{n}\right) P^{\prime}\left(\lambda_{n}\right)} d \lambda=\int_{-\sigma}^{\sigma} h_{n}(x) e^{i \lambda_{k} x} d x=\delta_{n, k} .
$$

Thus, by (3.2) and (4.5) we obtain

$$
\begin{aligned}
\int_{-\infty}^{\infty} F(\lambda) K_{N}(\lambda) d \lambda & =\sum_{n=-\infty}^{\infty} \sum_{k=-N}^{N} \frac{F\left(\lambda_{n}\right)}{P^{\prime}\left(\lambda_{k}\right)}\left(\frac{1}{2 \pi} \int_{-\infty}^{\infty} \frac{P(\lambda) B_{k}(\lambda)}{\left(\lambda-\lambda_{n}\right) P^{\prime}\left(\lambda_{n}\right)} d \lambda\right) \\
& =\sum_{n=-N}^{N} \frac{F\left(\lambda_{n}\right)}{P^{\prime}\left(\lambda_{n}\right)}=\sum_{n=-N}^{N}\left\langle f, g_{n}\right\rangle g_{n}^{*},
\end{aligned}
$$

where the last equality follows from (3.6). Interchanging the summation and the integration signs is possible by the dominated convergence theorem for Bochner integrals ([10], p. 35) since

$$
\int_{-\infty}^{\infty}\|F(\lambda)\|^{2} d \lambda \leq A^{-1} \sum_{n=-\infty}^{\infty}\left|\left\langle f, g_{n}\right\rangle\right|^{2} \int_{-\infty}^{\infty}\left|\frac{P(\lambda)}{\lambda-\lambda_{n}}\right|^{2} d \lambda \leq A^{-1} B D\|f\|^{2}<\infty .
$$

Taking the limit in (4.6) as $N \rightarrow \infty$ yields (4.1).

If the series $1 / 2 \pi \sum_{k=-\infty}^{\infty} B_{k}(\lambda) / P^{\prime}\left(\lambda_{k}\right)$ converges to a square integrable function $K(\lambda)$, then (4.1) becomes

$$
f=\int_{-\infty}^{\infty} F(\lambda) K(\lambda) d \lambda .
$$


In closing, we would like to recall that we have pointed out in Sec. 3 that the sampling points $\left\{\lambda_{k}\right\}$ do not have to be eigenvalues of a boundary-value problem. It is worth noting that it is not yet known if the points

$$
\lambda_{n}= \begin{cases}a n+b, & n=0,1,2, \ldots, \\ a n+c, & n=-1,-2, \ldots,\end{cases}
$$

where $a>0, b \neq c$, are eigenvalues of any boundary-value problem; however, a Kramer-type sampling theorem using these points as sampling points has recently been obtained by the author and it will appear in a separate publication.

\section{REFERENCES}

[1] J. Benedetto, Frame decompositions, sampling and uncertainty principle inequalities, Wavelets: Mathematics and Applications, Editors, J. Benedetto and M. Frazier, CRC Press, Boca Raton, FL (1994), pp. 247-304. MR 94i:94005

[2] Irregular sampling and frames, Wavelet-A Tutorial, Editor C. Chui, Academic Press (1991), pp. 1-63. MR 93c:42030

[3] J. Benedetto and W. Heller, Irregular sampling and the theory of frames I, Note di Matematica, Vol. X, Suppl. 1 (1990), pp. 103-125. MR 94e:94006

[4] F. Beutler, Error-free recovery of signals from irregularly spaced samples, SIAM Rev. 8 (1966), pp. 328-335. MR 34:5568

[5] _ Sampling theorems and bases in a Hilbert space, Information and Control, 4 (1961), pp. 97-117. MR 26:4832

[6] R. Boas, Entire Functions, Academic Press, New York (1954). MR 16:914f

[7] C. Heil and D. Walnut, Continuous and discrete wavelet transforms, SIAM Rev., Vol. 31 (1989), pp. 628-666. MR 91c:42032

[8] H. Kramer, A generalized sampling theorem, J. Math. Phys., 63 (1959/60), pp. 68-72. MR 21:2550

[9] N. Levinson, Gap and Density Theorem, Amer. Math. Soc. Colloq. Publs., Ser., Vol. 26, Amer. Math. Soc., Providence, RI (1940). MR 2:180d

[10] J. Mikusinski, The Bochner integral, Birkäuser, Basel (1978). MR 58:11296

[11] R. Paley and N. Wiener, Fourier transforms in the complex domain, Amer. Math. Soc. Colloq. Publs., Ser., Vol. 19, Amer. Math. Soc., Providence, RI (1934). MR 31:5038

[12] C. E. Shannon, A mathematical theory of communication, Bell System Tech. Report Journal, Vol. 27 (1948), pp. 379-423. MR 10:133e

[13] K. Yao, Applications of reproducing kernel Hilbert space of band-limited signal models, Information and Control, Vol. 11 (1967), pp. 429-444.

[14] A. I. Zayed, Advances in Shannon's sampling theory, CRC Press, Boca Raton, FL (1993). MR 95f:94008

[15] _ A new role of Green's function in interpolation and sampling theory, J. Math. Anal. Appl., 175 (1993), pp. 272-238. MR 94d:41001

Department of Mathematics, University of Central Florida, Orlando, Florida 32816

E-mail address: fdzayed@ucf1vm.cc.ucf.edu 